\title{
Development of a full-waveform voltage and current recording device for multichannel transient electromagnetic transmitters
}

\author{
Xinyue Zhang, Qisheng Zhang, Meng Wang, Qiang Kong, Shengquan Zhang, Ruihao He, Shenghui Liu, Shuhan Li, \\ and Zhenzhong Yuan
}

School of Geophysics and Information Technology, China University of Geosciences (Beijing), Beijing, 100083, China

Correspondence to: Qisheng Zhang (zqs@cugb.edu.cn)

Received: 8 March 2017 - Discussion started: 16 May 2017

Revised: 13 September 2017 - Accepted: 20 October 2017 - Published: 21 November 2017

\begin{abstract}
Due to the pressing demand for metallic ore exploration technology in China, several new technologies are being employed in the relevant exploration instruments. In addition to possessing the high resolution of the traditional transient electromagnetic method, high-efficiency measurements, and a short measurement time, the multichannel transient electromagnetic method (MTEM) technology can also sensitively determine the characteristics of a low-resistivity geologic body, without being affected by the terrain. Besides, the MTEM technology also solves the critical, existing interference problem in electrical exploration technology. This study develops a full-waveform voltage and current recording device for MTEM transmitters. After continuous acquisition and storage of the large, pseudo-random current signals emitted by the MTEM transmitter, these signals are then convoluted with the signals collected by the receiver to obtain the earth's impulse response. In this paper, the overall design of the full-waveform recording apparatus, including the hardware and upper-computer software designs, the software interface display, and the results of field test, is discussed in detail.
\end{abstract}

\section{Introduction}

The full-waveform voltage and current recording device of a multichannel transient electromagnetic method (MTEM) transmitter is an important component of MTEM exploration equipment. This device is responsible for the continuous acquisition and storage of the large pseudo-random current signals emitted by the MTEM transmitter. These signals are then convoluted with the signals collected by the receiver to ob- tain the impulse response of the ground under test (Zhong and Xue, 2014; Xue et al., 2015). Electrical methods (including conductive and inductive electrical methods) are crucial in metallic ore exploration. The conductivity, dielectric constant, and magnetic permeability of the rock ore are the main physical parameters measured in electrical geological exploration (Xue et al., 2007; Newman, 1989). Currently, the most critical problem with these methods is the interference from the area being explored. Solutions to reduce the effects of interference include increasing the transmission power of the field source, expanding the distance between the transmitter and receiver (conductive electrical methods), and reducing the observation signal frequency (inductive electrical methods). However, these measures often result in insignificant improvements in the signal-to-noise ratio (SNR) and higher costs. Therefore, they cannot satisfy the demands of the metallic ore exploration industry (Wright et al., 2001). The device examined in this study uses pseudo-random code to transmit certain artificial signals; the induced electromagnetic signals are then collected using a receiver at the remote end. Coherent decoding can significantly enhance the SNR of the acquired data and improve the exploration outcome; hence, it is suitable for monitoring high-power signals. Furthermore, the device also uses related technology to ensure the accuracy of the acquired signal amplitude and acquisition time, thereby achieving simultaneous acquisition of the high- and low-speed voltage and current.

In the future, the full-waveform voltage and current recording device can be used for marine exploration, marine electromagnetic exploration as a supplement to seismic exploration technology, can directly detect oil and gas in the structure of oil and gas, its status in the field of marine ex- 


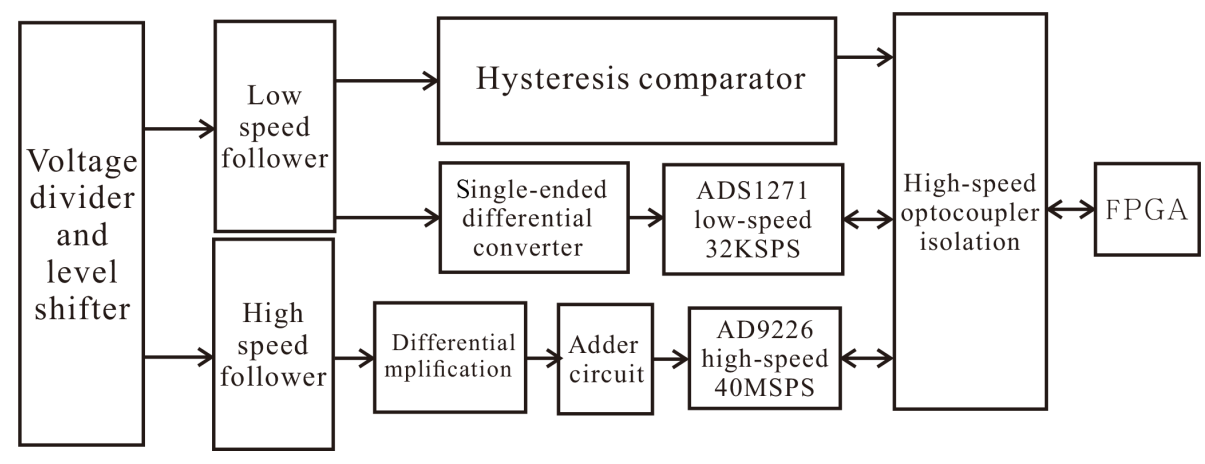

Figure 1. Block diagram of the conditioning process for voltage acquisition.

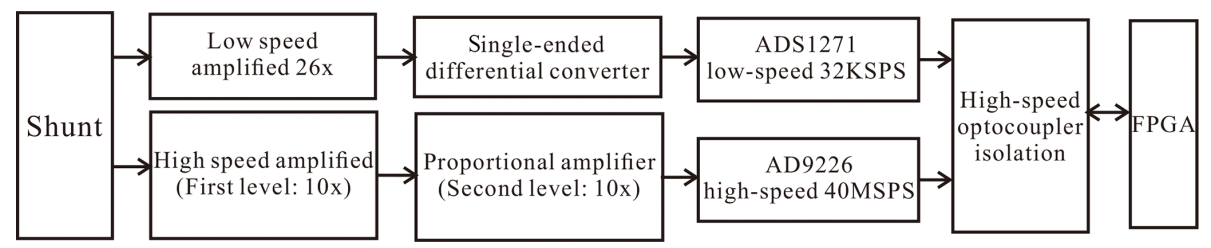

Figure 2. Block diagram of the conditioning process for current acquisition.

ploration is increasing. MTEM is accompanied by marine electromagnetic exploration came into being, if this technology is applied to land and sea oil and gas exploration, will greatly reduce the risk of three-dimensional seismic exploration, non-seismic methods of oil and gas resources exploration will have a very positive impact. In the future, the fullwaveform voltage and current recording device will be used for marine electromagnetic exploration as a supplement to seismic exploration technology. The device can directly detect oil and gas; its status in the field of marine electromagnetic exploration is increasing. If this technology can be applied to land and sea oil and gas exploration, it will greatly reduce the risk of three-dimensional seismic exploration. Additionally, non-seismic methods of oil and gas resources exploration will have a very positive impact.

This study discusses the development of the full-waveform voltage and current recording device for MTEM transmitters. The article is structured as follows: Sect. 2 presents the hardware circuit design of the full-waveform recording device, Sect. 3 describes the upper-computer software design, and Sect. 4 presents the test results.

\section{Hardware circuit design of the full-waveform recording devices}

The hardware circuit design of the full-waveform recording device for MTEM transmitters can be divided into the voltage and current channel designs (Ziolkowski et al., 2007). The design principles are depicted in Figs. 1 and 2. The voltage channel divides the input signal into two levels: large range and small range. For large-range levels, the maxi- mum input for the transmitter voltage amplitude is $1000 \mathrm{~V}$, whereas, when adjusted to the small-range level, the maximum input is $100 \mathrm{~V}$; relays are used to switch between the large- and small-range levels. As the maximum voltage amplitude emitted by the transmitter is $1000 \mathrm{~V}$, a voltagereducing follower is needed before being input to the analogto-digital converter (ADC) for conversion (Boris and Bernhard, 2003). The input end of the low-speed ADC is a differential converter; hence, the single-ended signal outputs from the follower should be converted into differential signals. However, as the input end of the high-speed ADC is single ended, only an adder circuit is needed. In addition, In order to achieve frequency measurement, we use a hysteresis comparison circuit, where LM311 plays an important part (Jia et al., 2009). LM311 is a low-speed operational amplifier which is exclusively used in voltage comparison; the typical response time is $200 \mathrm{~ns}$. We can use a single supply voltage or a dual power supply.

The calculation formula for high hysteresis comparison voltage is as follows:

$V_{\mathrm{inH}}=\frac{R 4}{R 4+R 2}\left(V_{\mathrm{OH}}-V_{\mathrm{ref}}\right)+V_{\mathrm{ref}}$.

The calculation formula for low hysteresis comparison voltage is as follows:

$V_{\text {inL }}=\frac{R 4}{R 4+R 2}\left(V_{\mathrm{OL}}-V_{\text {ref }}\right)+V_{\text {ref }}$.

The current channel differs from the voltage channel in that it does not require repeated frequency measurements; hence, a hysteresis comparator is not required. The large current 


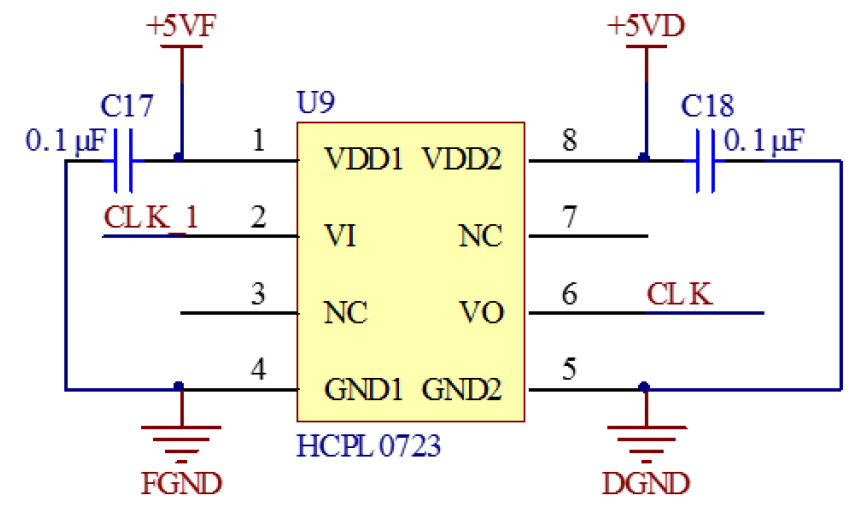

Figure 3. Schematic circuit diagram of high-speed optocoupler isolation.

signals from the transmitter need to undergo a voltage-tocurrent conversion, which is achieved using a shunt. Hence, an amplifier circuit with a high bandwidth and high amplification magnitude is required. The other parts of the conditioning circuit are similar to those of the voltage acquisition.

The signals emitted by the transmitter are strong with large voltages and currents, whereas those of the master FPGA are weak (Huang et al., 2015). Hence, an isolation barrier is needed between the two for protecting the master circuit and the upper computer connected to it. Therefore, we designed a high-speed optocoupler isolation circuit. Every digital inputoutput of the ADC on the acquisition board requires a highspeed optocoupler for isolation; the input and output directions of the optocoupler were verified one-by-one to ensure circuit correctness (Jhin et al., 2014). HCPL0723 is a highspeed, positive logic CMOS optocoupler of Avago Technologies company, which supports a transmission speed of up to $50 \mathrm{Mbit}$; the maximum transmission delay is $22 \mathrm{~ns}$, and the external circuit connection is simple, requiring only the additional coupling capacitor at both ends of the power supply, as shown in Fig. 3. In contrast to traditional optocouplers, the photodiode control pin of HCPL0723 has no flow current, and the output logic pins do not need to be pulled up. Instead, the current that drives the photodiode flows from the input pin (1 pin).

The acquisition circuit of the full-waveform recording device can be divided into low speed and high speed. The lowspeed acquisition circuit uses the ADC ADS1271 to achieve a sampling rate of $32 \mathrm{kSPS}$. A high-precision 24-bit $\Delta \Sigma$ type ADC was used, supporting the following modes: high speed, high precision, and low power (Li et al., 2013). Under the high-speed mode, the sampling rate can reach a maximum of $105 \mathrm{kSPS}$. In the high-precision mode, the SNR can reach $109 \mathrm{dBm}$ and in the low-power mode the overall power consumption is $35 \mathrm{~mW}$ (Texas Instruments, 2007). For highspeed acquisition, ADC AD9226 was used to achieve a sampling rate of $40 \mathrm{MSPS}$; this is a 12-bit pipeline ADC with a maximum sampling rate of 65 MSPS and a bandwidth of

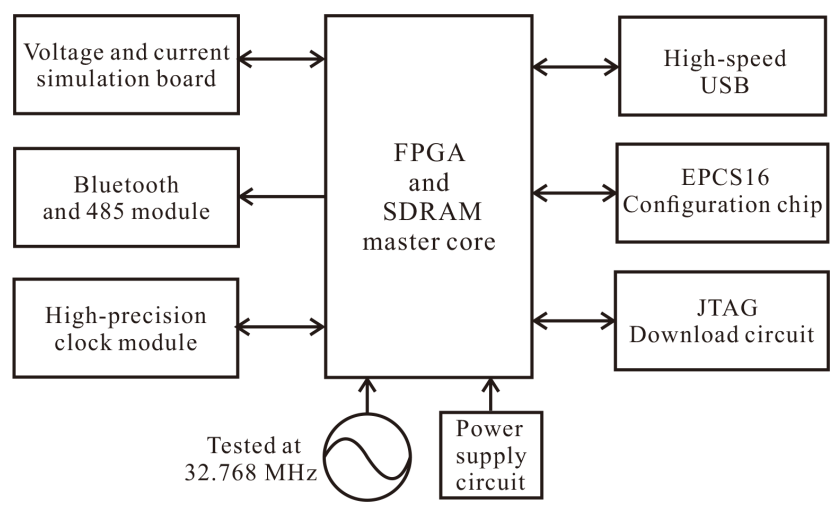

Figure 4. Block diagram of the FPGA master circuit.

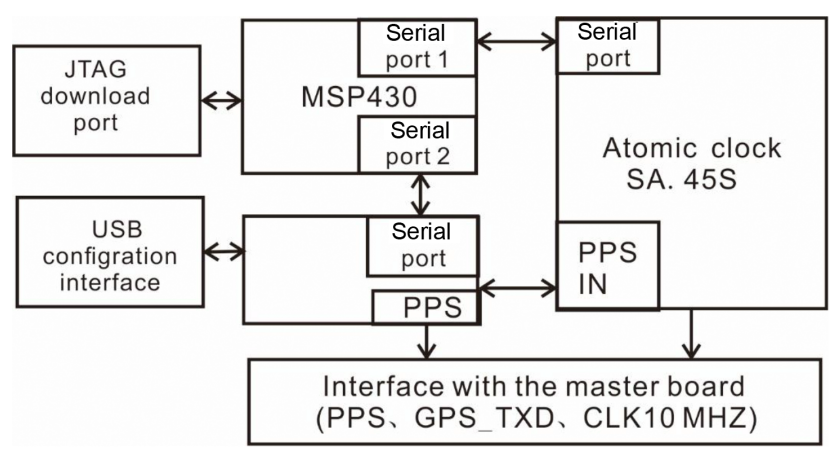

Figure 5. Block diagram of high precision atomic clock circuit.

up to $750 \mathrm{MHz}$. For an input signal of $31 \mathrm{MHz}$, the spuriousfree dynamic range is $85 \mathrm{dM}$ (Analog Devices Incorporated, 2001).

The FPGA master circuit is the control core of the MTEM transmitter full-waveform voltage and current recording (Sun et al., 2016). It is responsible for the core functions of the recording device, including control acquisition and data transfer (Ziolkowski et al., 2011). The master circuit is mainly composed of the FPGA and a few external storage devices; its block diagram is depicted in Fig. 4. The storage devices include an SRAM, SDRAM, and serial and parallel flashes. Among them, the SDRAM is used as the data buffer for low-speed acquisition and the parallel flash, as the memory for the FPGA-configuration firmware. In order to ensure data integrity in the low-speed acquisition, an external SDRAM was used as a buffer for the data from this circuit. The 256 Mbit single-clock SDRAM used in this study has four groups of internal banks. Each bank has 4 trillion stored words and each word has a 16-bit length; i.e., the data bus is composed of D0-D15 lines. The $80 \mathrm{MHz}$ clock for the SDRAM is provided by the FPGA to satisfy the low-speed acquisition data rate. The master circuit uses high-speed USB integrated circuits (ICs) to implement the USB transfer protocol, which is simple and convenient. The FPGA of the master circuit uses a JTAG chain to burn the SRAM configuration 


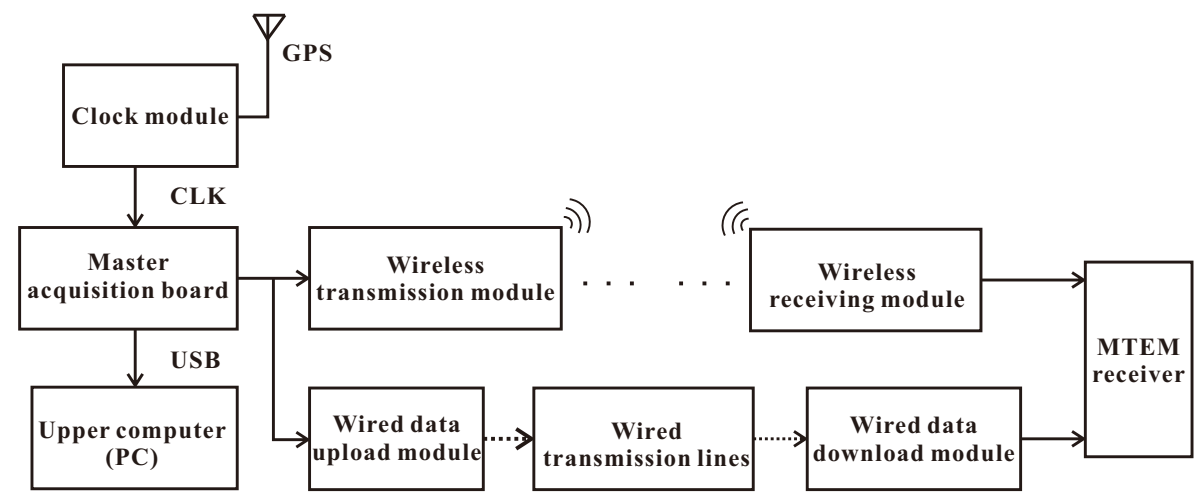

Figure 6. Block diagram of the Bluetooth and 485 module transmission.

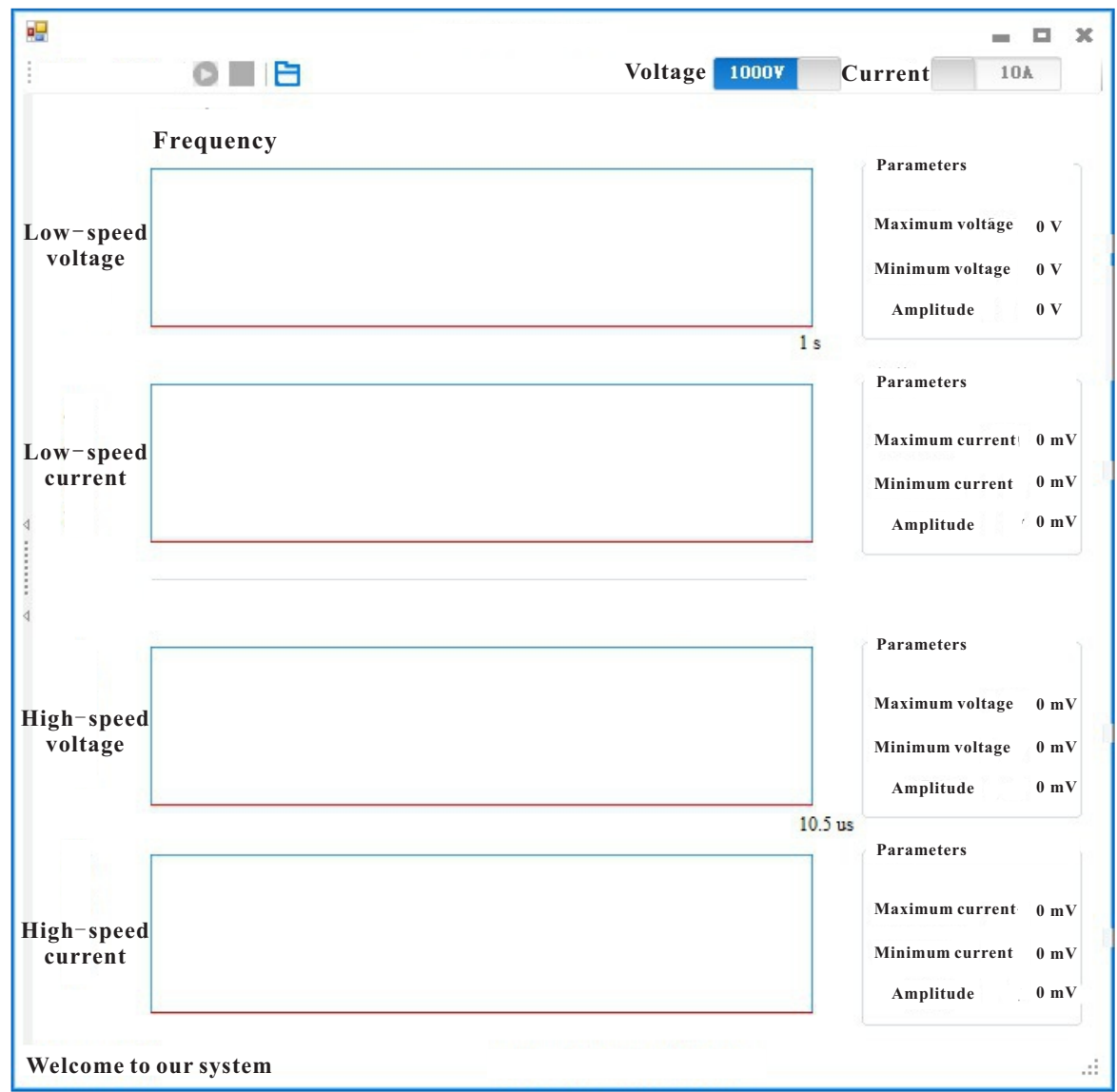

Figure 7. Interface display of the main window form on the upper computer.

file onto the serial flash, EPCS16. After the FPGA is powered up again, in the active serial mode, it reads the configuration file in the external serial flash internally and completes the IC configuration process.

After the upper computer receives the transmission signal waveform, it calculates the voltage and current peak values, frequency, timestamp, and the other transmission waveform information (Zhong et al., 2016). This information is then transferred to the lower computer via the command control channel. The lower-computer forwards these data, as per the serial communication protocol, to the 485 and Bluetooth modules; the data are then transferred to a remote PC via wired or wireless methods. Figure 6 shows the process block diagram.

The MTEM method requires the simultaneous recording of the transmitter current and receiver voltage signals fol- 
(a)

Voltage (V) - sample number

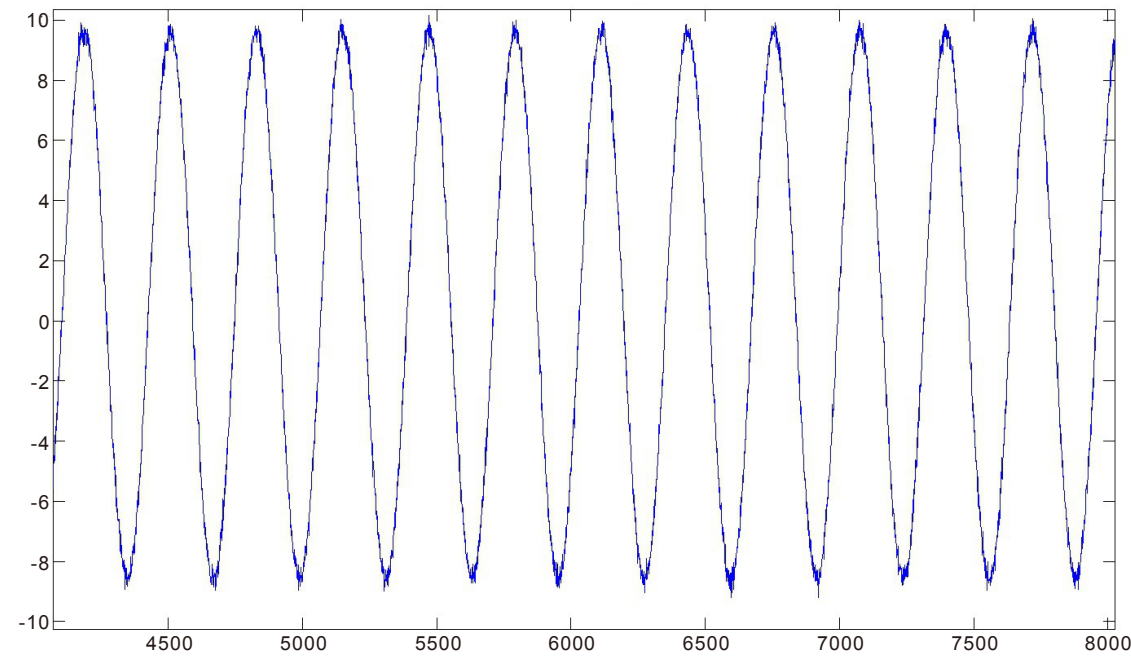

(b) Amplitude (dB) - frequency (Hz)

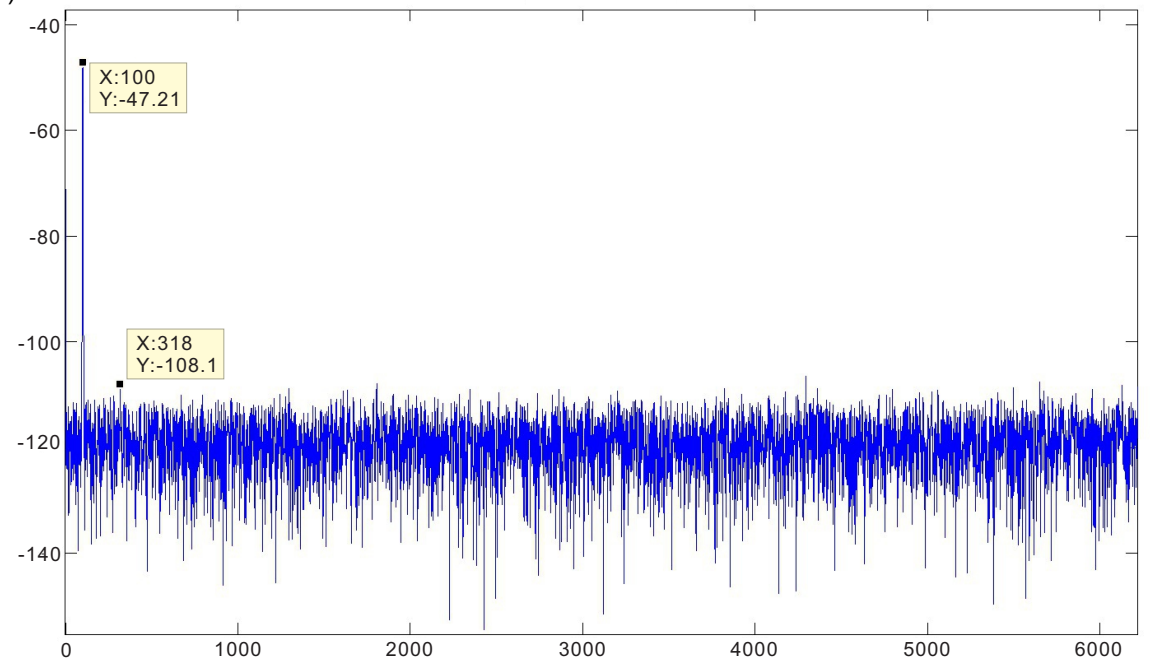

Figure 8. Test results of the low-speed voltage acquisition channel. (a) Time domain waveform of the $20 \mathrm{Vpp}, 100 \mathrm{~Hz}$ sinusoid acquired by the recording device at a $1000 \mathrm{~V}$ level. (b) Amplitude-frequency characteristics of the 32000 samples subjected to FFT (Hanning window truncation).

lowed by the convolution of the two to calculate the earth's impulse response. Hence, the acquisition time for these two signals is an important parameter (Wright, 2003). This design uses an atomic clock as the clock source for the master circuit and all the ADC clocks are based on stable clock frequency dividers or multipliers of the atomic clock outputs (Xu et al., 2004; Zhang et al., 2015). By synchronizing the pulse per second (PPS) of the atomic clock and the GPS, and then synchronizing the PPS of the receiver and GPS, we could approximate the simultaneous acquisition of the two acquisition systems (Olalekan and Di, 2015).
The block diagram of the high-precision atomic clock circuit is shown in Fig. 5. The clock board consists of three parts: the MSP430 microcontroller, GPS module, and atomic clock module. In addition, the clock board also includes some necessary download ports, configuration interface, and the interface with the main control board.

The MSP430 microcontroller, the master, downloads the program through the JTAG download port. The microcontroller communicates with the atomic clock SA.45S through serial port 1 , receives the serial data to obtain the atomic clock running status, and sends the serial port command to 
(a)
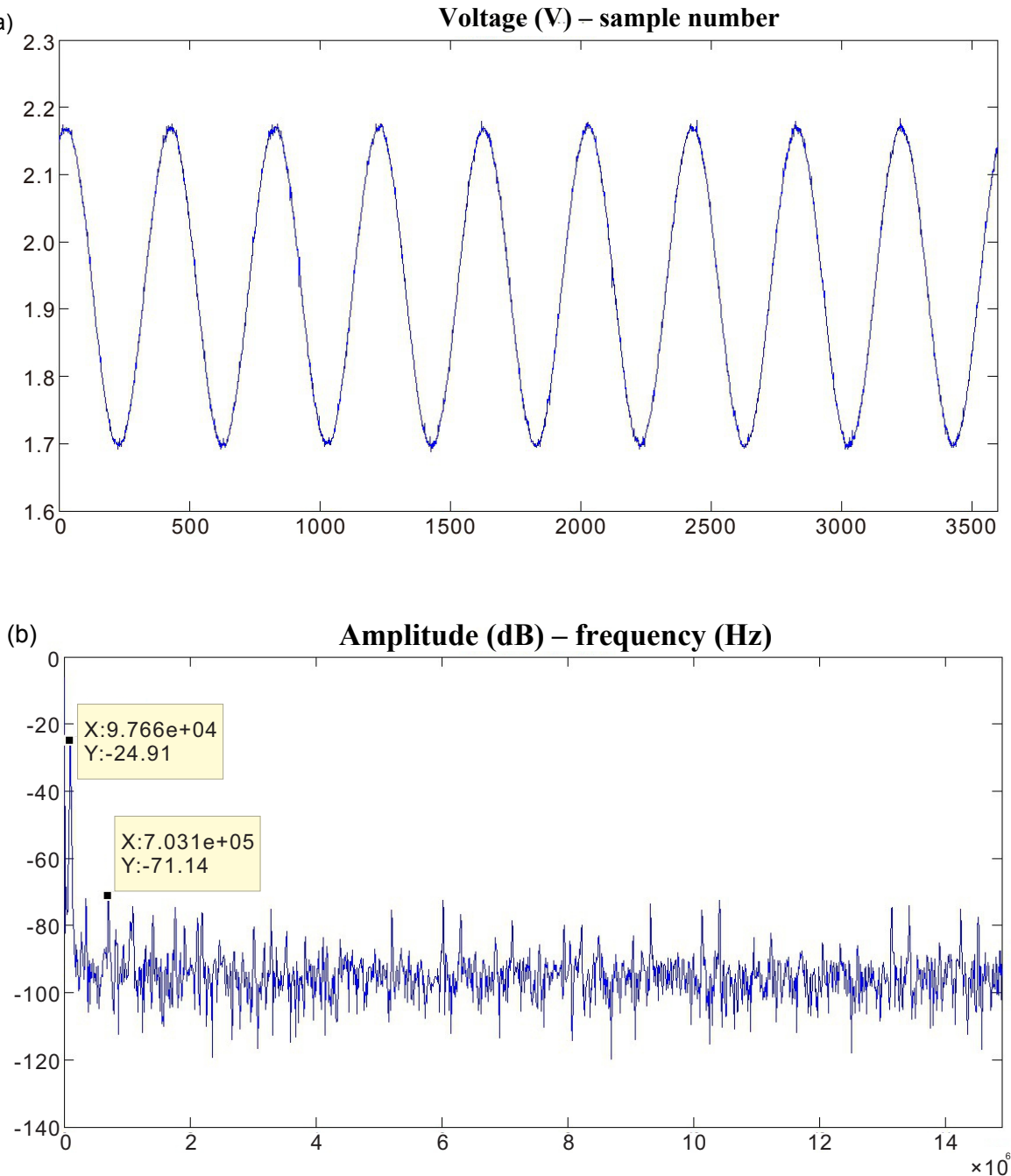

Figure 9. Test results of the high-speed voltage acquisition channel. (a) Time domain waveform of the $20 \mathrm{Vpp}, 100 \mathrm{kHz}$ sinusoid acquired by the high-speed voltage recording device at a $100 \mathrm{~V}$ level. (b) Amplitude-frequency characteristics of the 4906 samples subjected to FFT (Hanning window truncation).

control its taming time. MSP430 and GPS module communicate with each other through serial port 2.

The transmitter of the GPS serial port is also connected to the input-output of the FPGA so that the acquisition data contains the location coordinate information. The GPS second pulse signal is connected to the atomic clock of the PPS_IN pin to tame the atomic clock module. GPS sets the mode of operation through its full-speed USB interface; after setting the work mode of host computer software, you can store the work mode information in the internal of GPS, so that the host computer can automatically read the last saved work mode information from the internal of GPS when the module power was cut off.

\section{Upper-computer software design}

The upper-computer program was built on the Visual Studio platform using $\mathrm{C \#}$ Windows forms. The program mainly includes the main window form (Form1), the sub-window form (Form2), and a self-defined class function. The program framework is described below: in the main window form, the read and write functions of each channel were assigned to eight corresponding threads. Sampling or interception was performed on the data flags and channel types to obtain data points for plotting the waveform; four drawing threads were opened to plot the waveforms. Class functions were formed by packaging each channel of the USB equipment into a class, based on the endpoint address. Each class included the endpoint parameters and two methods to read (using the asynchronous read mode) and write data into the files. The read and write methods were assigned to the threads of their 

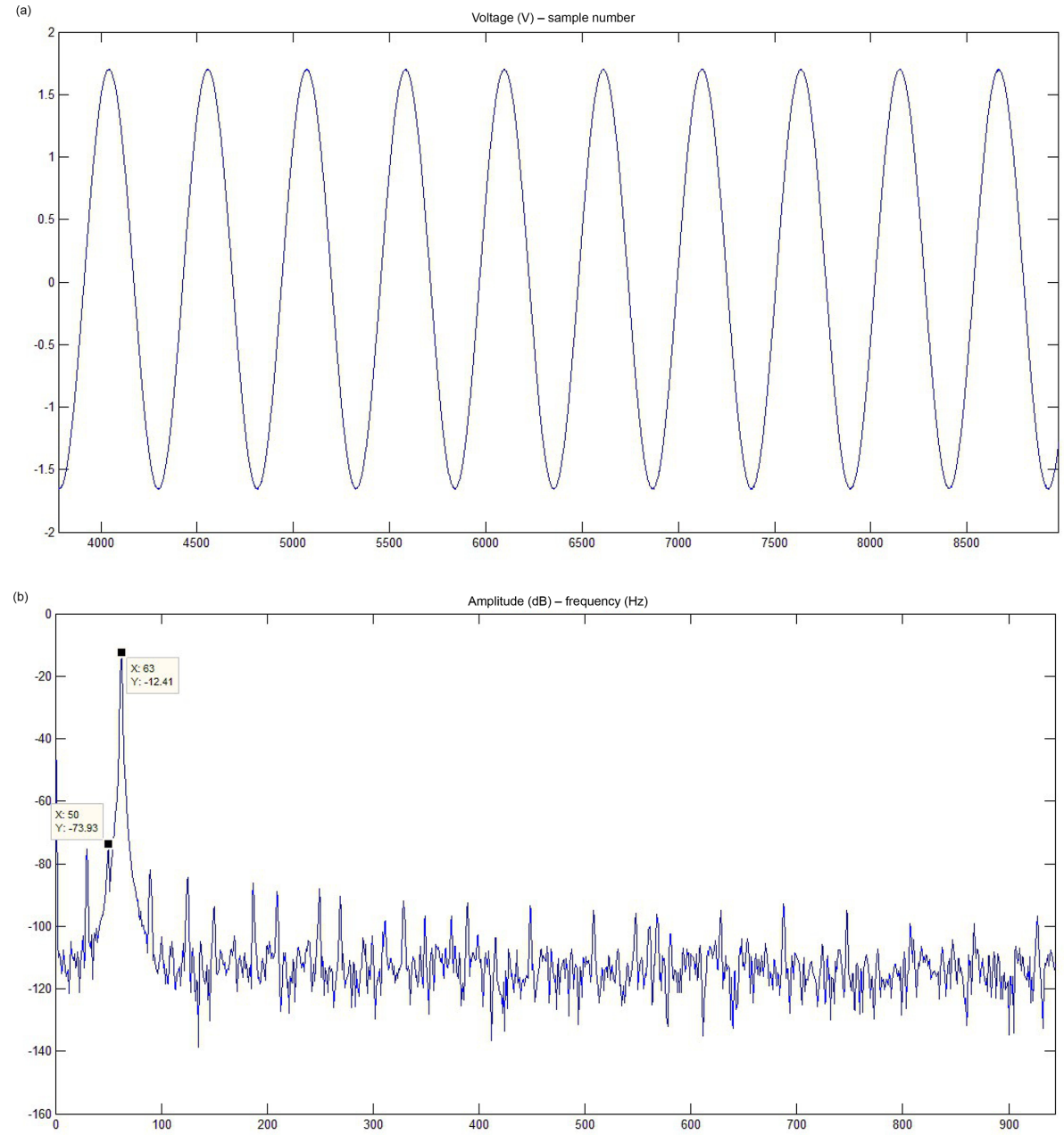

Figure 10. Test results of the low-speed current acquisition channel. (a) Time domain waveform of the $150 \mathrm{mVpp}, 62.5 \mathrm{~Hz}$ sinusoid acquired by the low-speed current recording device at a 50 A level. (b) Amplitude-frequency characteristics of the 32000 samples subjected to FFT (Hanning window truncation).

own channels, in the main function. The sub-window form was used to open and review the stored file data. The entire software uses the Metro style, which provides a more humanistic human-computer interface. Figure 7 displays the main window form.

\section{Test results of the full-waveform recording device}

Overall performance testing was conducted after developing the full-waveform recording device. For the low-speed voltage, $20 \mathrm{Vp}, 100 \mathrm{~Hz}$ sinusoidal signals produced by a signal generator were fed directly to the $1000 \mathrm{~V}$ high-voltage input end. Using a voltage-regulated power supply at a sampling rate of $32 \mathrm{kSPS}, 32000$ samples were acquired; further, fast Fourier transform (FFT) was performed on these samples and MATLAB was used to generate the time and frequency domain waveforms. For the high-speed voltage, the acquisition performance at the $100 \mathrm{~V}$ level was tested. $20 \mathrm{Vp}, 100 \mathrm{kHz}$ sinusoidal signals from a signal generator were fed directly to the high-speed voltage input end and the device was powered by a lithium battery (Wang et al., 2015). 4096 samples were acquired at a sampling rate of 40 MSPS; FFT was then performed on these samples and MATLAB was also used to generate the time and frequency domain waveforms. When testing the low-speed current acquisition board, the level was shifted to $50 \mathrm{~A} ; 150 \mathrm{mVpp}, 62.5 \mathrm{~Hz}$ sinusoidal signals from a signal generator were fed directly to the low-speed current input end. Further, MATLAB was used to generate the time and frequency domain waveforms. For testing the highspeed current acquisition board, the level was shifted to $50 \mathrm{~A}$; $150 \mathrm{mVpp}, 100 \mathrm{KHz}$ sinusoidal signals from a signal generator were fed simultaneously to the high-speed and low-speed current input ends. Then, MATLAB was used to generate the time and frequency domain waveforms (Zhang et al., 2016). 

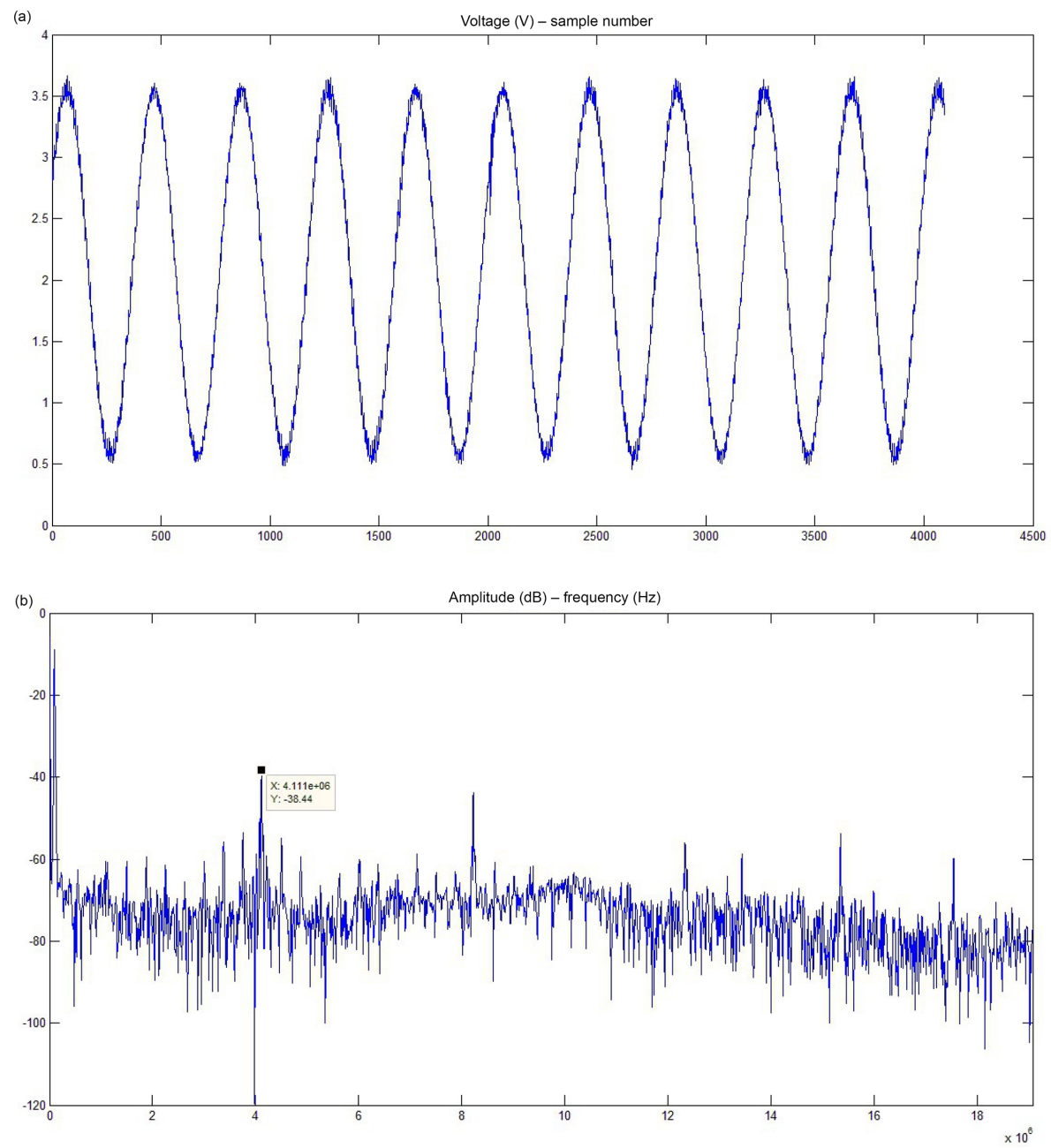

Figure 11. Test results of the high-speed current acquisition channel. (a) Time domain waveform of the $150 \mathrm{mVpp}, 100 \mathrm{KHz}$ sinusoid acquired by the high-speed current recording device at a $50 \mathrm{~A}$ level. (b) Amplitude-frequency characteristics of the 4906 samples subjected to FFT (Hanning window truncation).

\subsection{Low-speed voltage channel}

Figure 8 shows the test results of the low-speed voltage acquisition channel. Figure $8 \mathrm{a}$ is the time domain waveform of the sinusoid acquired by the recording device, whereas Fig. $6 \mathrm{~b}$ is the frequency domain waveform. As seen from Fig. 8 b, the corresponding amplitude of the input $20 \mathrm{Vpp}$ sinusoidal signal was $-42 \mathrm{dBFS}$; i.e., the full amplitude was approximately $2000 \mathrm{Vpp}$. The SNR at this point was also higher than $50 \mathrm{~dB}$; hence, the overall SNR was higher than $90 \mathrm{~dB}$. A low-speed acquisition precision of up to $\pm 1 \%$ was attained.

\subsection{High-speed voltage channel}

Figure 9 displays the test results of the high-speed voltage acquisition channel. In Fig. 9b, harmonic distortion at an amplitude of approximately $-71 \mathrm{~dB}$ is observed; hence, its am- plitude input was approximately $200 \mathrm{Vpp}$ (200 Vpp is equivalent to $-20 \mathrm{~dB}$ ). Therefore, the overall SNR was higher than $60 \mathrm{~dB}$. A high-speed acquisition precision of up to $\pm 1 \%$ was attained.

\subsection{Low-speed current channel}

Figure 10 shows the test results of the low-speed current acquisition channel. We can see from the amplitude-frequency characteristics that there was a $50 \mathrm{~Hz}$ power frequency interference at $-73 \mathrm{~dB}$. Relatively, many harmonic components were present; the average noise threshold was lower than $-100 \mathrm{~dB}$ and its SNR higher than $60 \mathrm{~dB}$.

\subsection{High-speed current channel}

Figure 11 displays the test results of the high-speed current acquisition channel. 


\section{Conclusion}

In this study, the development of a full-waveform voltage and current recording device for MTEM transmitters was presented. First, the hardware circuit was designed to complete the pre-conditioning and acquisition of the signals, which were then transferred to the FPGA for data processing. Then, the upper-computer software was designed to further process the data and present them as graphic plots. The overall performance was tested, with individual tests for the high- and low-speed channels for the voltage and current. The maximum transmission current and voltage acquired by the device were $50 \mathrm{~A}$ and $1000 \mathrm{~V}$, respectively; a $\pm 1 \%$ sampling precision was attained for both current and voltage. This full-waveform recording device can be used to monitor the high-power, full-waveform voltages and currents of MTEM transmitters. This recording device has higher precision, finer edge details, lower noise, and other advantages. Hence, it can be used for real-time recording and transmission to the receiver for coherent demodulation.

Data availability. No data sets were used in this article.

Competing interests. The authors declare that they have no conflict of interest.

Acknowledgements. This work was supported by the Fundamental Research Funds for the Central Universities of China (no. 2652015213), the National Natural Science Foundation of China (no. 41574131), and the National Major Scientific Research Equipment Research Projects of China (no. ZDYZ2012-1-05-01).

Edited by: Luis Vazquez

Reviewed by: three anonymous referees

\section{References}

Analog Devices Incorporated: AD9226 datasheet, Analog Devices Incorporated, USA, 2001.

Boris, M. and Bernhard, E. B.: A 12-bit 75-MS/s Pipelined ADC Using Open-Loop Residue Amplification, IEEE J. Solid-State Circuits, 12, 2040-2050, 2003.

Huang, Y. W., Yan, J. H., and Zhou, W.: Data Acquisition Test System Based on FPGA, Modern Radar, 37, 73-76, 2015.

Jhin, F. H., Jin, Y. W., and Cheng, K. H.: An 8-bit 20 MS/s Successive Approximation Register Analog-to-digital Converter with Low Input Capacitance, Int. J. Eng. Pract. Res., 4, 83-88, 2014.

Jia, H. Y., Chen, G. C., and Zhang, H.: A new structure of substage in pipelined analog-to-digital converters, The Journal of China Universities of Posts and Telecommunications, 16, 86-90, 2009.

Li, X. Y., Liu, M. C., Sun, Z. L., Xiao, L. Y., and He, S. Y.: Effect of ionizing radiation on dual 8-bit analog-to-digital converters
(AD9058) with various dose rates and bias conditions, Chinese Phys. B, 22, 633-637, 2013.

Newman, G. A.: Deep transient electromagnetic soundings with a grounded source over near-surface conductors, Geophys. J., 98, 587-601, 1989.

Olalekan, F. and Di, Q.: Multi-transient electromagnetic (MTEM) response modeling using finite difference time domain method, International Workshop and Gravity, Electrical \& Magnetic Methods and their Applications, Chenghu, China, 107-110, April 2015

Sun, Y. M., Zhang, Y. F., Wang, M. C., and Wang, X. C.: Design of FPGA-Based Real-Time Radar Signal Acquisition and Display System, Chin. J. Electron Devices, 3, 639-643, 2016.

Texas Instruments Incorporated:, ADS1271 datasheet, Texas Instruments Incorporated, Texas, USA, 2007.

Wang, X. L., Gao, J. X., Tian, J. S., and Zhang, Y. T.: Design of power transformer in MTEM transmitter power, Atlantis Press, 161-164, https://doi.org/10.2991/cmes-15.2015.47, 2015.

Wright, D. A.: Detection of hydrocarbons and their movement in a reservoir using time-lapse multi-transient electromagnetic MTEM data [D], University of Edinburgh, Edinburgh, 2003.

Wright, D. A., Ziolkowski, A., and Hobbs, B. A.: Hydrocarbon detection with a multi-channel transient electromagnetic survey[C], Proceedings of the 71st Annual International Meeting, SEG, Expanded Abstracts, San Antonio, USA, 1435-1438, 2001.

Xu, J. T., Yao, S. Y., Zhao, Y. Q., Zhang, W., Li, S. R., and Zhang, S. C.: Optimization of Power Dissipation in Pipelined Analog-toDigital Converter, Transactions of Tianjin University, 10, 280 284, 2004.

Xue, G. Q., Li, X., and Di, Q. Y.: The progress of TEM in theory and application, Prog. Geophys., 22, 1195-1200, 2007.

Xue, G. Q., Yan, S., Di, Q. Y., Li, X., Wang, R., Li, H., and Zhong, H. S.: Technical analysis of multi-transient electromagnetic method, J. Earth Sci. Environ., 37, 94-100, 2015.

Zhang, W. W., Di, Q. Y., and Lei, D.: Estimation of the earth impulse response of MTEM in very noisy environment, International Conference on Environment and Engineering Geophysics \& Summit Forum of Chinese Academy of Engineering on Engineering Science and Technology, 26-29 June 2016, Beijing, China, 2016.

Zhang, Y. Q., Xing, W. W., and Zhang, C. X.: A rapid weak signal detection approach in resonant pressure sensors; Signal detection for optical AC and DC voltage sensors based on pockels effect, The 4th International Conference on Mechatronics, Materials, Chemistry and Computer Engineering, 12-13 December 2015, Xian, China, 2651-2654, 2015.

Zhong, H. S. and Xue, G. Q.: Application of MTEM Method, Annual Meeting of Chinese Geoscience Union, 1399, 2014.

Zhong, H. S., Xue, G. Q., Li, X., Zhi, Q. Q., and Di, Q. Y.: Pseudo wavefield extraction in the multi-channel transient electromagnetic (MTEM) method, Chin. J. Geophys., 59, 4424-4431, 2016.

Ziolkowski, A., Hobbs, B. A., and Wright, D.: Multi-transient electromagnetic demonstration survey in France, Geophysics, 72, F197-F209, 2007.

Ziolkowski, A., Parr, R., Wright, D., Nockles, V., Limond, C., Morris, E., and Linfoot, J.: Multi-transient electromagnetic repeatability experiment over the North Sea Harding field, Geophys. Prospect., 58, 1159-1176, 2011. 